\title{
Hidroxilação dos óleos de girassol, milho e crambe e caracterização química dos polióis vegetais obtidos
}

\author{
Hydroxylation of sunflower, corn and crambe oils \\ and chemical characterization of the obtained \\ vegetable polyols
}

Bárbara Zon Nascimento ${ }^{1}$, Ana Paula Oliveira Costa ${ }^{1}$

\begin{abstract}
${ }^{1}$ Universidade do Federal Espírito Santo, Campus São Mateus, Rodovia BR 101 Norte, km 60, Bairro Litorâneo, CEP: 29932-540, São Mateus, Espírito Santo, ES, Brasil.

e-mail: barbazon@hotmail.com, ana.o.costa@ufes.br
\end{abstract}

\section{RESUMO}

Neste trabalho foram sintetizados e caracterizados polióis vegetais a partir do óleo de girassol, milho e crambe. Os polióis foram obtidos a partir da modificação química dos óleos vegetais pela reação de hidroxilação direta com o sistema perácido in situ (ácido fórmico e peróxido de hidrogênio) na proporção molar (óleo/ácido/peróxido) de 1,0:3,0:1,5, temperatura de $85^{\circ} \mathrm{C}$, tempo de reação de $6 \mathrm{~h}$ com agitação mecânica. Os produtos foram caracterizados quanto à viscosidade, densidade, espectroscopia na região do infravermelho (FTIR), índice de hidroxila (IOH), índice de iodo (II) e acidez (IA). O poliol com as melhores propriedades foi obtido a partir do óleo de girassol, com IOH de 72,88 mg de KOH/g de amostra, II de 11,87 g $\mathrm{de}_{2} / \mathrm{g}$ de amostra, IA de 16,13 mg de $\mathrm{NaOH} / \mathrm{g}$ de amostra e viscosidade de 1096,4 mPa.s. O poliol de crambe apresentou valores de IOH de 40,80 mg de KOH/g de amostra, II de 78,18 g de I/2/g de amostra, IA de 4,25 mg de NaOH/g de amostra e viscosidade de 649,9 mPa.s. Por fim, o poliol de milho apresentou IOH de 37,40 mg de KOH/g de amostra, II de $62,47 \mathrm{~g}$ de $\mathrm{I}_{2} / \mathrm{g}$ de amostra, IA de 54,62 mg de NaOH/g de amostra e viscosidade de 486,8 mPa.s. A aplicação da técnica de FTIR nos polióis vegetais obtidos permitiu a análise qualitativa dos grupos hidroxilas pelo aparecimento de uma banda alargada na faixa de 3400-3500 $\mathrm{cm}^{-1}$, característica desse grupo funcional. Foi possível sintetizar os polióis vegetais a partir da reação de hidroxilação dos óleos estudados e os produtos apresentam potencial para serem usados na síntese de poliuretanos flexíveis, aumentando-se o valor agregado das matérias-primas estudadas.

Palavras-chave: Oleoquímica, polióis vegetais, hidroxilação.

\section{ABSTRACT}

In this work, vegetable polyols were synthesized and characterization from sunflower's, corn's and crambe's oils. The polyols were obtained from the chemical modification of the vegetable oils by the direct hydroxylation reaction with the peracid system in situ (formic acid and hydrogen peroxide) in the molar ratio (oil/acid/peroxide) of 1,0:3.0:1,5, temperature of $85^{\circ} \mathrm{C}$, reaction time of $6 \mathrm{~h}$ with mechanical agitation. The products were characterized by viscosity, density, infrared spectroscopy (FTIR), hydroxyl number (IOH), iodine value (II) and acidity level (IA). The polyol with the best properties was obtained from sunflower oil, with IOH of $72.88 \mathrm{mg} \mathrm{KOH} / \mathrm{g}$ of sample, II of $11.87 \mathrm{~g} \mathrm{I} / \mathrm{g}$ sample, IA of $16.13 \mathrm{mg}$ of $\mathrm{NaOH} / \mathrm{g}$ sample and viscosity of $1096.4 \mathrm{mPa} . \mathrm{s}$. The crambe polyol presented IOH of $40.80 \mathrm{mg} \mathrm{KOH} / \mathrm{g}$ of sample, II of $78.18 \mathrm{~g}$ of $\mathrm{I}_{2} / \mathrm{g}$ sample, IA of $4.25 \mathrm{mg}$ of $\mathrm{NaOH} / \mathrm{g}$ of sample and viscosity of $649.9 \mathrm{mPa}$.s. Finally, the corn polyol presented $\mathrm{IOH}$ of $37.40 \mathrm{mg}$ of $\mathrm{KOH} / \mathrm{g}$ of sample, II of $62.47 \mathrm{~g}$ of $\mathrm{I}_{2} / \mathrm{g}$ of sample, IA of $54.62 \mathrm{mg}$ of NaOH$/ \mathrm{g}$ of sample and viscosity of $486.8 \mathrm{mPa}$.s. The application of the FTIR technique in the obtained polyols allowed the qualitative analysis of the hydroxyl groups by the appearance of a wide band in the range of $3400-3500 \mathrm{~cm}^{-1}$, characteristic of this functional group. It was possible to synthesize the vegetable polyols from the hydroxylation reaction of the studied oils and the products have potential to be used in the synthesis of flexible polyurethanes, increasing the added value of the raw materials studied.

Keywords: Oilchemistry, vegetable polyols, hydroxylation. 


\section{INTRODUÇÃO}

A indústria química tem buscado novos materiais provenientes de fontes renováveis, visando produtos biodegradáveis, obtidos por processos com baixo custo de energia e não poluentes [1].

Nesse contexto, os óleos vegetais se apresentam como matérias-primas viáveis, pois são uma das fontes renováveis mais abundantes no planeta, disponíveis em grande quantidade, diversificados e com menor custo, constituindo assim, uma alternativa que apresenta vantagens econômicas, energéticas e ambientais em relação as matérias-primas obtidas por derivados do petróleo [2].

Embora os óleos vegetais sejam usados há muito tempo pela indústria química, atualmente existe um maior interesse na utilização destes quando modificados quimicamente, pois podem ser utilizados como matéria-prima para a produção de polímeros [3].

Estudos têm mostrado que as insaturações constituem os sítios ativos para reações químicas, logo, quanto maior o número de insaturações, maior a probabilidade das reações acontecerem com maior eficácia [4]. Por este motivo, a análise comparativa da composição em termos de ácidos graxos é fundamental para a escolha da matéria-prima que será utilizada.

A Tabela 1 apresenta a composição dos principais ácidos graxos presentes nos óleos de girassol (Helianthus annuus L), de milho (Zea mays) e de crambe (Crambe abyssinica Hochst). É possível observar que no óleo de crambe predomina o ácido erúcico, um ácido graxo com 22 carbonos e uma ligação dupla, já nos óleos de milho e de girassol, predomina o ácido linoléico, um ácido graxo que tem cadeia menor, com 18 carbonos, porém é mais insaturado, contendo 2 ligações duplas.

Tabela 1: Composição dos principais ácidos graxos (\% em massa) no óleo de girassol, milho e crambe.

\begin{tabular}{l|c|c|c}
\hline \multicolumn{3}{|c}{ ÓLEO (\% EM MASSA) } \\
\hline ÁCIDO GRAXO & GIRASSOL & MILHO & CRAMBE \\
\hline Palmítico (C16:0) & --- & 11,0 & 2,0 \\
\hline Palmitoleico (C16:1) & 6,1 & --- & -- \\
\hline Esteárico (C18:0) & 3,3 & 2,0 & --- \\
\hline Oleico (C18:1) & 16,9 & 29,0 & 19,0 \\
\hline Linoleico (C18:2) & 73,7 & 57,0 & 9,0 \\
\hline Linolênico (C18:3) & --- & 0,7 & -- \\
\hline Aracdínico (C20:0) & --- & 0,3 & 2,0 \\
\hline Erúcico (C22:1) & --- & --- & 59,0 \\
\hline
\end{tabular}

(Fonte: Adaptada de REIZNAUTT et al., 2009 [5], ZLATANIC et al., 2004 [6], TAVARES et al., 2017 [7]).

As ligações duplas presentes nos triacilglicerídeos dos óleos vegetais podem ser funcionalizadas através da inserção de grupos hidroxilas para síntese de polióis vegetais [8]. Os polióis são matérias-primas utilizadas na produção de poliuretanas (PUs), que são polímeros versáteis com uma gama de aplicações [9]. Esses polímeros são utilizados na indústria automotiva, calçadista, construção civil, fabricantes de móveis, embalagens, revestimentos, tintas, adesivos e espumas [10, $11,12,13]$.

Muitos esforços vêm sendo realizados com o intuito de substituir os polióis petroquímicos por polióis obtidos a partir da modificação química de óleos vegetais, obtendo-se polímeros "ecologicamente corretos". Nesse sentido, os polióis oleoquímicos se destacam como matérias-primas alternativas para a síntese de produtos uretânicos, cujas propriedades competem com a de PUs produzidas a partir de polióis petroquímicos [14, 15].

Os métodos mais estudados para a síntese de polióis à base de óleo são a hidroxilação das ligações duplas carbono-carbono, a alcoólise para se obter mono e diglicéridos [10], a reação de epoxidação, seguida da abertura do anel epóxi de óleos vegetais [16], hidroformilação [17], ozonólise [4] e transesterificação [8].

Nesse contexto, a reação de hidroxilação de óleos vegetais para obtenção de polióis tem sido objeto de estudo de muitos pesquisadores, a seguir são destacados alguns trabalhos. AUNG et al. [18] investigaram a síntese de um poliol a partir do óleo de pinhão-manso por duas rotas químicas: hidroxilação seguida de alcoólise ou epoxidação. Os polióis obtidos foram tratados com diisocianato para produção de adesivos de poliuretanas (PU). Os polióis sintetizados por alcoólise apresentaram índice de hidroxila ( $\mathrm{IOH}$ ) de $153 \mathrm{mg} \mathrm{KOH} / \mathrm{g}$ e índice de acidez (IA) de 5,79 mg KOH/g, enquanto que os epoxidados obtiveram IOH de $171 \mathrm{mg} \mathrm{KOH} / \mathrm{g}$ e IA de 8,19 mg KOH/g. Os adesivos de PU sintetizados utilizando os polióis vegetais mostraram boas propriedades físico-químicas exigidas para esse tipo de aplicação, de modo que essa 
matéria-prima renovável é considerada um substituto para os materiais não-renováveis existentes na produção de adesivos.

ALLAUDDIN et al. [4] sintetizaram um poliol a partir do óleo de soja por epoxidação e hidroxilação subsequente, utilizando-o para produzir revestimentos de poliuretano/uréia. O poliol de soja (PS) apresentou um índice de hidroxila (IOH) de 231,3 mg KOH/g, índice de iodo (II) de 1,22 g de I/g, índice de acidez (IA) de 1,15 mg KOH/g e viscosidade de $43750 \mathrm{mPa}$.s. O PS de alta funcionalidade foi reagido com diferentes isocianatos e secos em umidade atmosférica para obter os revestimentos PU-Ureia. Todos os revestimentos mostraram excelente resistência térmica, mecânica e solvente. Verificou-se que a funcionalidade da estrutura de polióis e isocianatos pode variar para obtenção de revestimentos com características desejáveis, o que levou os autores a conclusão de que os polióis oleoquímicos podem ser um valioso substituto para os atuais materiais à base de petróleo em segmentos de revestimento de alto desempenho.

LI et al. [3] estudaram a metátese do óleo de soja para síntese de polióis pelas reações de epoxidação e hidroxilação. Os polióis obtidos apresentaram valores de IOH entre 83 e $263 \mathrm{mg} \mathrm{KOH} / \mathrm{g}$ e foram usados para fazer espumas de poliuretano com combinações controladas de força (tensão a $10 \%$ de deformação de 0,07 a $0,75 \mathrm{MPa}$ ) e flexibilidade adequada para aplicações versáteis, particularmente aquelas que exigem resistência e resiliência. Pelos dados de resistência à compressão e recuperação, os autores concluíram que os polióis oleoquímicos podem ser usados para a preparação de espumas flexíveis e semi-rígidas excelentes.

VERONESE et al. [19] modificaram o óleo de soja por meio da reação de hidroxilação, seguida de transesterificação com um álcool polifuncional para aumentar a funcionalidade $\mathrm{OH}$. O óleo de mamona, que já possui grupos hidroxila em sua estrutura molecular, foi apenas transesterificado. Após a modificação química dos óleos estudados, os polióis apresentaram valores de IOH entre 393 e $477 \mathrm{mg} \mathrm{KOH} / \mathrm{g}$ óleo, além de baixa viscosidade e peso molecular. Dessa forma, a partir dos polióis sintetizados, foi possível obter uma espuma vegetal rígida com densidade aparente de $50 \pm 1 \mathrm{~kg} / \mathrm{m}^{3}$ e resistência à compressão em torno de $200 \mathrm{kPa}$, de modo que essa apresentou propriedades semelhantes a uma espuma comercial usada para aplicações de isolamento térmico.

COSTA et al. [14] sintetizaram compósitos de poliuretanas utilizando um poliol vegetal com IOH de $190 \mathrm{mg} \mathrm{KOH/g}$ obtido a partir da hidroxilação do óleo de soja com uma proporção molar de 1,0:3,0:1,5 (óleo:ácido:peróxido), por 4 h à 65 ${ }^{\circ} \mathrm{C}$. Utilizou-se a razão molar $[\mathrm{OH}] /[\mathrm{NCO}]$ de 0,9 e a mistura foi curada por $24 \mathrm{~h}$ a $65^{\circ} \mathrm{C}$. Na preparação dos compósitos foram usadas as mesmas razões molares e as condições de cura empregadas na PU. No entanto, variou-se as quantidades das cargas de 1 a 15\% em peso das argilas: Campo do Tenente, Pitanga, Rio do Rastro e Sílica. Os ensaios de flexão mostraram que a adição das cargas tende a aumentar o valor do módulo de elasticidade de 124 para a PU até $142 \mathrm{MPa}$. A densidade de reticulação variou de $150 \mathrm{~mol} / \mathrm{m}^{3}$ para a PU até $176 \mathrm{~mol} / \mathrm{m}^{3}$ e a densidade das amostras variaram de 0,86 a $0,911 \mathrm{~g} / \mathrm{cm}^{3}$. A estabilidade térmica foi de $210^{\circ} \mathrm{C}$ e a adição de carga não acarretou mudanças. Os valores da dureza variaram de 72 a 78 . O trabalho permitiu concluir que foi possível obter poliuretanas elastoméricas a partir do poliol vegetal sintetizado.

NASCIMENTO et al. [20] sintetizaram um poliol vegetal a partir do óleo de crambe por meio da reação de hidroxilação com um sistema a base de ácido perfórmico "in situ" e peróxido de hidrogênio, avaliando os seguintes parâmetros reacionais: tempo, temperatura, proporção molar e agitação. A melhor condição reacional foi alcançada na proporção molar de 1,0:3,0:1,5 (óleo:ácido:peróxido), por $6 \mathrm{~h}$, a $85^{\circ} \mathrm{C}$, sob agitação mecânica, em que obteve-se o maior grau de hidroxilação com o valor de $\mathrm{IOH}$ de 40,55 mg de $\mathrm{KOH} / \mathrm{g}$ de óleo, gerando assim um produto de maior valor agregado.

Cada rota de síntese apresenta vantagens e desvantagens que são inerentes ao processo, de modo que o poliol sintetizado apresenta características distintas, podendo obter tanto polióis com alta funcionalidade do grupo hidroxila (grande número de radicais hidroxilas introduzidos na cadeia) ou polióis com conversão parcial, ou seja, baixa conversão de duplas ligações com poucos radicais hidroxilas introduzidos na cadeia [21]. Sendo assim, há várias possibilidades de formulações obtendo vários polióis, cuja escolha dependerá de sua aplicação [14].

Este trabalho tem como objetivo realizar a hidroxilação direta de espécies vegetais pouco exploradas na área de obtenção de novos materiais, tais como: os óleos de crambe, milho e girassol, bem como, a caracterização de todos os polióis sintetizados quanto à viscosidade, densidade, índice de hidroxila (IOH), índice de iodo (II), índice de acidez (IA) e espectroscopia na região do infravermelho (FTIR). 


\section{MATERIAIS E MÉTODOS}

\subsection{Materiais}

Os óleos de girassol (OG) e milho (OM) foram adquiridos no mercado local, ambos da Marca Liza (São Mateus, Espírito Santo, Brasil). O óleo de crambe (OC) foi doado pela Fundação MS.

\subsection{Métodos}

\subsubsection{Síntese e tratamento dos polióis vegetais}

Para a síntese dos polióis, o sistema reacional utilizado teve como proporção molar óleo/ácido/peróxido (1,0:3,0:1,5), temperatura de $85^{\circ} \mathrm{C}$, tempo de reação de 6 h e agitação mecânica.

$\mathrm{O}$ ácido fórmico foi adicionado ao sistema em temperatura ambiente e o peróxido de hidrogênio em banho de gelo. Em seguida, o sistema foi colocado em um banho de aquecimento, na temperatura de $85^{\circ} \mathrm{C}$, por $6 \mathrm{~h}$ sob agitação mecânica. Na Figura 1 está apresentado o aparato experimental utilizado na síntese.

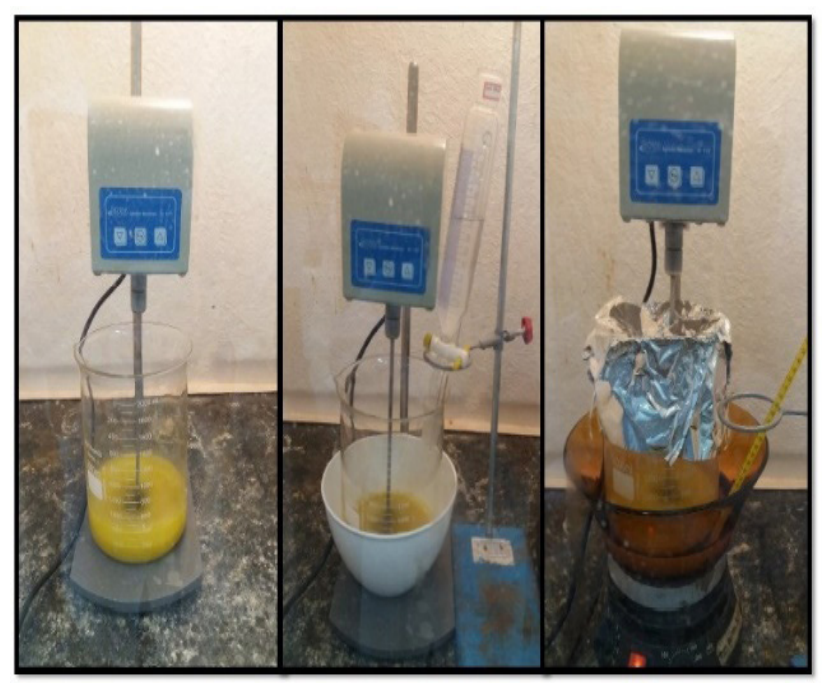

Figura 1: Aparato experimental para reação de hidroxilação do óleo vegetal.

Ao final do tempo de reação, os produtos foram tratados até a neutralização e secos. A etapa de neutralização foi feita com a adição de soluções de carbonato de sódio $\left(\mathrm{Na}_{2} \mathrm{CO}_{3(\mathrm{aq})}\right)$ a $10 \%$ e cloreto de sódio $\left(\mathrm{NaCl}_{(\mathrm{aq})}\right)$ a $30 \%$ até atingir $\mathrm{pH}$ neutro. $\mathrm{Na}$ etapa de secagem foi adicionado sulfato de sódio anidro $\left(\mathrm{Na}_{2} \mathrm{SO}_{4}\right)$, este foi mantido por $24 \mathrm{~h}$, após este período, o agente secante foi retirado por filtração a vácuo. Por fim, o poliol foi submetido a etapa final para evaporação de solvente adicional na temperatura de $60{ }^{\circ} \mathrm{C}$ sob agitação magnética. Na Figura 2 estão ilustradas as etapas do tratamento dos polióis. 


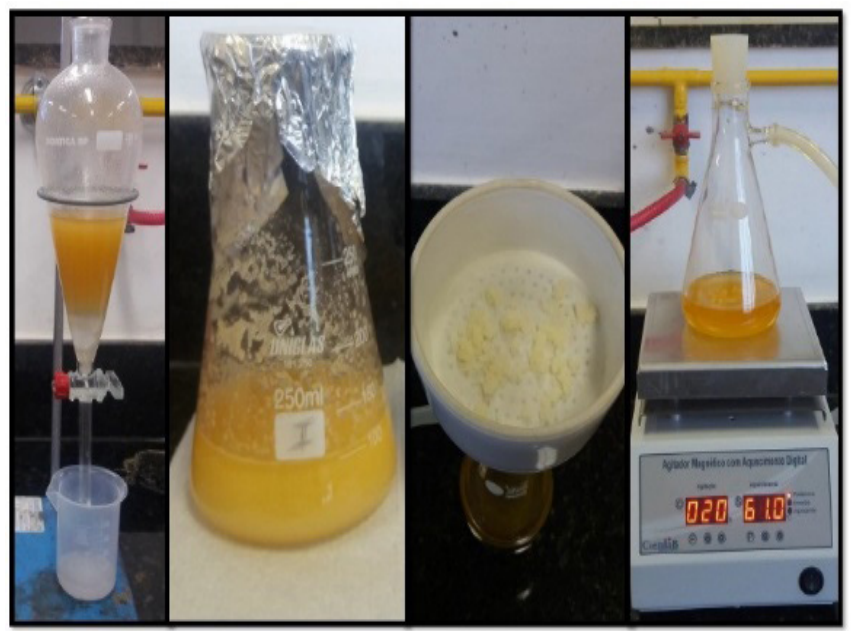

Figura 2: Etapas de tratamento dos polióis vegetais.

\subsubsection{Caracterização dos óleos e polióis vegetais}

Os óleos de girassol (OG), milho (OM) e crambe (OC) foram caracterizados pelos índices de acidez (IA), índice de iodo (II), densidade, viscosidade e espectroscopia na região do infravermelho (FTIR).

Os polióis vegetais foram caracterizados pelos índices de acidez (IA), índice de iodo (II), índice de hidroxila (IOH), densidade, viscosidade e espectroscopia na região do infravermelho (FTIR).

\subsubsection{1 Índice de acidez (IA)}

O índice de acidez é definido como o número de miligramas de hidróxido de sódio $(\mathrm{NaOH})$ necessários para neutralizar os ácidos livres presentes em um grama de óleo ou gordura. Tal índice foi determinado segundo a metodologia estabelecida pela AOCS por meio da norma Ca 3a-63.

\subsubsection{2 Índice de iodo (II)}

O índice de iodo é uma medida do grau de insaturação dos ácidos graxos presentes em um óleo ou gordura. Este índice é definido como a quantidade em gramas de iodo absorvidos por $100 \mathrm{~g}$ de gordura ou óleo. Tal índice foi determinado segundo a metodologia estabelecida pela AOCS por meio da norma Cd 1-25.

\subsubsection{3 Índice de hidroxila (IOH)}

O índice de hidroxila $(\mathrm{IOH})$ é uma medida do conteúdo de hidroxila $(\mathrm{OH})$ no poliol e é definido em miligrama de hidróxido de potássio por grama de amostra. Tal índice foi determinado segundo a metodologia estabelecida pela AOCS por meio da norma Cd 13-60.

\subsubsection{Densidade}

A densidade foi determinada por picnometria, utilizando uma balança analítica (Shimadzu, modelo AY220) e um picnômetro de $25 \mathrm{~mL}$, na temperatura ambiente. A correlação entre temperatura da água e a densidade da água foi feita por meio da tabela contida no Handbook of Chemistry and Physics [22]. O procedimento foi realizado em triplicata.

\subsubsection{Viscosidade}

A viscosidade foi obtida usando o viscosímetro rotacional digital MVD-5, marca Marte Científica, modelo 860A21. O instrumento é equipado com cilindros de diâmetros diferentes (spindles), em que é utilizado o cilindro adequado conforme a viscosidade do fluido. Para este trabalho, o spindle de referência L1 foi usado para as medidas. O viscosímetro foi acoplado a um banho termostático na temperatura de $25^{\circ} \mathrm{C}$. O procedimento foi realizado em triplicata. 


\subsubsection{Espectroscopia de infravermelho (FTIR)}

Para a realização das análises de espectroscopia de infravermelho por Transformada de Fourier (FTIR) foi utilizado o Cary 630 FTIR Spectrometer da Agilent Technolologies em modo Diamond ATR com resolução de 0,05 $\mathrm{cm}^{-1}$, abrangendo a região de 4000 a $400 \mathrm{~cm}^{-1}$, com varredura de 200 scan, em que foram feitos filmes usando cristais de brometo de potássio $(\mathrm{KBr})$.

\section{RESULTADOS E DISCUSSÃO}

Os polióis sintetizados a partir dos óleos de girassol, milho e crambe foram tratados e caracterizados quanto aos índices de acidez (IA), índice de iodo (II), índice de hidroxila (IOH), densidade e viscosidade. As análises de IA, II, densidade e viscosidade serviram para estabelecer uma comparação entre o óleo refinado e o produto obtido a partir da reação de hidroxilação. O IOH foi utilizado para definir uma possível aplicação do poliol. Os dados experimentais referentes à caracterização dos óleos vegetais e dos polióis de girassol (PG), milho (PM) e crambe (PC) são mostrados na Tabela 2.

Tabela 2: Resultados experimentais da caracterização dos óleos vegetais e polióis.

\begin{tabular}{|c|c|c|c|c|c|}
\hline AMOSTRAS & $\begin{array}{c}\text { IA } \\
\text { (mg de } \mathrm{NaOH} / \mathrm{g} \\
\text { de amostra) }\end{array}$ & $\begin{array}{c}\text { II } \\
\text { (g de } \mathrm{I}_{2} / \mathrm{g} \text { de } \\
\text { amostra) }\end{array}$ & $\begin{array}{c}\mathrm{IOH} \\
\text { (mg de } \mathrm{KOH} / \mathrm{g} \\
\text { de amostra) }\end{array}$ & $\begin{array}{c}\text { DENSIDADE } \\
\left(\mathrm{g} / \mathrm{cm}^{3}\right)\end{array}$ & $\begin{array}{l}\text { VISCOSIDADE } \\
\text { (mPa.s) }\end{array}$ \\
\hline $\mathrm{OG}$ & 0,72 & 135,11 & --- & $0,919 \pm 0,001$ & $82,6 \pm 0,2$ \\
\hline PG & 16,13 & 11,87 & 72,88 & $0,998 \pm 0,001$ & $1096,4 \pm 0,2$ \\
\hline $\mathrm{OM}$ & 0,67 & 129,2 & --- & $0,917 \pm 0,001$ & $100,4 \pm 0,1$ \\
\hline PM & 54,62 & 62,47 & 37,40 & $0,961 \pm 0,001$ & $486,8 \pm 0,8$ \\
\hline $\mathrm{OC}$ & 4,23 & 99,33 & --- & $0,913 \pm 0,001$ & $150,6 \pm 0,1$ \\
\hline $\mathrm{PC}$ & 4,25 & 78,18 & 40,80 & $0,929 \pm 0,001$ & $649,9 \pm 0,9$ \\
\hline
\end{tabular}

Legenda: Índice de acidez (IA), índice de iodo (II), índice de hidroxila (IOH), óleo de girassol (OG), poliol de girassol (PG), óleo de milho (OM), poliol de milho (PM), óleo de crambe (OC) e poliol de crambe (PC).

Por meio dos dados apresentados na Tabela 2, pode-se observar que o óleo de crambe (OC) é o que apresenta maior acidez e viscosidade, e menor densidade e insaturação. O óleo de girassol (OG) é o mais insaturado, mais denso e menos viscoso. O óleo de milho $(\mathrm{OM})$ apresenta valores intermediários para as análises quando comparado às outras oleaginosas em estudo.

Os valores determinados para o índice de acidez (IA) dos óleos vegetais estão na mesma ordem de grandeza com relação aos valores citados na literatura, sendo que para o girassol pode variar entre 0,3 e $2 \mathrm{mg}$ de NaOH/g de amostra, para o milho entre 0,3 e 6 e o do crambe é encontrado na faixa de 2 à $5 \mathrm{mg}$ de $\mathrm{NaOH} / \mathrm{g}$ de amostra [23, 24].

Os índices de acidez dos polióis são relativamente maiores que o dos respectivos óleos vegetais, mesmo após a etapa de neutralização realizada no tratamento dos produtos. Tal fato pode ser decorrente da presença de ácido fórmico remanescente ou a formação de ácidos graxos livres provenientes da hidrólise do óleo. O poliol de milho (PM) revelou um alto índice de acidez, sugerindo a presença de ácidos graxos livres, comportamento semelhante foi observado no trabalho de SILVA [25], que também caracterizou polióis obtidos a partir do óleo de milho (OM).

Os valores do índice de iodo (II) encontrados experimentalmente estão de acordo com a composição dos óleos apresentada na Tabela 1, sendo que o óleo de girassol (OG) é o mais insaturado, ou seja, com maior II. Tal resultado pode ser justificado já que na composição desse óleo, o ácido linoleico está presente em maior quantidade e esse contém duas ligações duplas em sua estrutura química, promovendo maior insaturação no óleo de girassol (OG).

Os índices de iodo (II) dos óleos vegetais estão coerentes quando comparados aos encontrados em outras pesquisas, de modo que para o óleo de girassol (OG), este valor está na faixa entre 110 e $143 \mathrm{~g}$ de $\mathrm{I}_{2} / \mathrm{g}$ de amostra, para o milho encontra-se entre 107 e 135, e para o óleo de crambe (OC) varia de 90 à $100 \mathrm{~g} \mathrm{de} \mathrm{I}_{2} / \mathrm{g}$ de amostra [23, 26].

Os polióis apresentaram valores de índice de iodo (II) significativamente menores que os óleos vegetais de origem. Esse comportamento ocorre devido a eficiência da reação de hidroxilação, que se inicia pelo ataque do ácido à insaturação do óleo, com formação de um intermediário epóxido e subsequente abertura do anel epóxido para a formação de uma hidroxila e de um grupo formiato ligados à estrutura do triglicerídeo [27].

Apesar das condições reacionais serem as mesmas para os três óleos vegetais estudados, os polióis apresentaram 
conversões diferenciadas, uma vez que o consumo de insaturações, observado por meio da diminuição do II do poliol em relação ao respectivo óleo, ocorreu de forma peculiar para cada oleaginosa em análise. Tal comportamento propiciou maior IOH nos óleos que tiveram maior consumo de insaturações, conforme era esperado. De maneira análoga, visualiza-se no estudo de YAKUSHIN et al. [28], em que foram obtidos polióis à base de óleo de girassol, colza e mamona nas mesmas condições de síntese, e o IOH variou de maneira individual para cada óleo vegetal considerado na pesquisa.

Mediante a isso, nota-se que quanto maior o índice de insaturação do óleo vegetal, mais interessante essa matéria-prima é para a síntese de polióis, visto que, existem reações químicas para conversão das duplas ligações presentes na molécula do triacilglicerídeo, possibilitando a obtenção de polióis vegetais com estruturas variadas [29, 30].

A densidade é uma propriedade que está diretamente ligada com a estrutura molecular. Quanto maior o comprimento da cadeia carbônica, maior será a densidade, no entanto, esse valor decrescerá quanto maior for o número de insaturações presentes na molécula, sendo que as impurezas também podem influenciar no valor da densidade [31].

As densidades dos óleos determinadas experimentalmente estão de acordo com a literatura, em que se verifica valores entre 0,915 e $0,923 \mathrm{~g} / \mathrm{cm}^{3}$ para o óleo de girassol (OG), 0,914 à 0,925 para o óleo de milho $(\mathrm{OM})$ e 0,908 à $0,914 \mathrm{~g} / \mathrm{cm}^{3}$ para o óleo de crambe (OC) [23, 24].

Os polióis apresentaram valores de densidade maiores quando comparados as dos óleos de origem. Tal fato ocorre devido a diminuição do número de insaturações dos produtos pela entrada dos grupos hidroxilas, demonstrados pelo índice de iodo (II).

O ensaio reológico determinou a viscosidade dinâmica ou absoluta $(\mu)$ para os óleos vegetais, a viscosidade geralmente aumenta com o comprimento da cadeia e diminui com a quantidade de insaturações [32]. As duplas ligações provocam dobras nas cadeias carbônicas dos ácidos, dificultando a aproximação e aglomeração das moléculas, contribuindo para a redução da viscosidade [33]. Portanto, pode-se verificar que o óleo de crambe (OC) apresenta maior viscosidade devido ao maior tamanho da cadeia e menor quantidade de insaturações.

O aumento de viscosidade nos polióis é significativo quando comparado aos óleos, visto que os grupos hidroxilas $(\mathrm{OH})$ introduzidos na molécula levam ao aumento no número de ligações de hidrogênio, que são forças intermoleculares fortes, elevando significativamente a viscosidade do produto [6, 34].

A viscosidade está diretamente relacionada ao índice de hidroxilas ( $\mathrm{IOH})$ dos polióis que estão dispostos na Tabela 2. Pela análise dos dados, percebe-se que o óleo de girassol (OG) foi o mais hidroxilado por ser mais insaturado, sendo o poliol mais viscoso devido às ligações de hidrogênio presentes nos grupos hidroxilas.

O índice de hidroxila do poliol de milho (PM) é o menor, apresentando por consequência menor viscosidade, visto que, apesar de ser altamente poliinsaturado, o óleo de milho (OM) é bastante estável, pois contém altos níveis de antioxidantes naturais e baixa porcentagem de ácido linolênico (ácido graxo com 18 carbonos na cadeia e três insaturações), conforme apresentado na Tabela 1. A alta estabilidade do óleo de milho (OM) pode, ainda, estar parcialmente relacionada à distribuição não casual dos ácidos graxos nas moléculas dos triacilglicerídeos. A maior parte dos ácidos graxos insaturados encontra-se esterificada na posição 2 dos triacilglicerídeos que é menos reativa. Os ácidos graxos poliinsaturados estão, portanto, mais protegidos das reações oxidativas [35].

O poliol de crambe $(\mathrm{PC})$ teve o índice de hidroxila ( $\mathrm{IOH})$ intermediário em relação aos outros polióis vegetais, e o mesmo comportamento foi observado para o valor relativo a viscosidade determinada para este poliol.

Devido ao fato de que não há relatos na literatura para polióis obtidos a partir de óleo de milho e girassol, existe a dificuldade para realizar uma comparação entre os dados obtidos. Com relação ao poliol crambe, foi observado que o valor de IOH foi de 40,80 mg de KOH/g de amostra, semelhante ao trabalho de NASCIMENTO et al. [20] que sintetizaram um poliol a partir do mesmo óleo vegetal e obtiveram um valor bem próximo de $\mathrm{IOH}$ de 40,55 mg de KOH/g de óleo.

De acordo com CARRIÇO [36], o índice de hidroxila é o parâmetro utilizado para definir a aplicação de um poliol. Os polióis que originam as espumas flexíveis e elastômeros apresentam $\mathrm{IOH}$ entre 28 e $160 \mathrm{mg}$ de $\mathrm{KOH} / \mathrm{g}$, enquanto para espumas rígidas são requeridos polióis com IOH de 250 a $1000 \mathrm{mg}$ de KOH/g. Dessa forma, os polióis vegetais sintetizados nesse trabalho têm potencial para aplicação na produção de espumas flexíveis e elastômeros, uma vez que os valores de $\mathrm{IOH}$ determinados experimentalmente se encontram na faixa entre 37,40 e 72,88 $\mathrm{mg}$ de $\mathrm{KOH} / \mathrm{g}$.

A presença dos grupos hidroxilas $(\mathrm{OH})$ pode ser detectada qualitativamente pela análise do espectro na região do infravermelho dos polióis em comparação com os óleos de origem. Os gráficos referentes ao infravermelho estão apresentados nas Figuras 3, 4 e 5. 


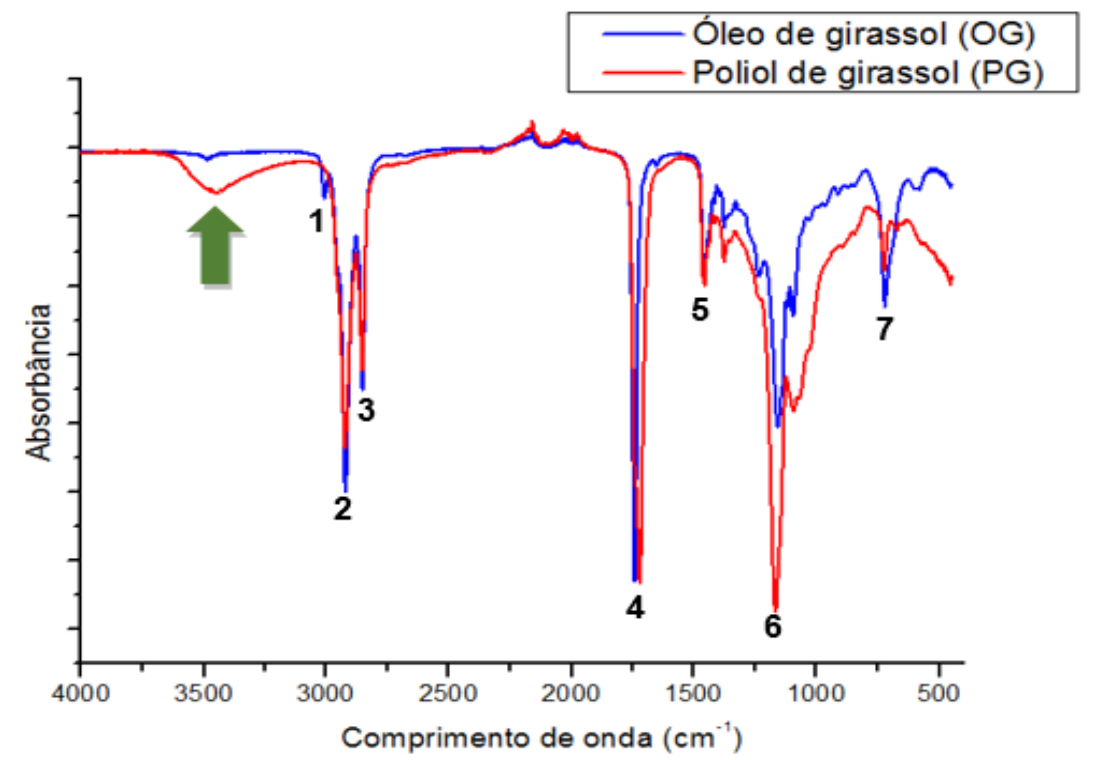

Figura 3: Espectro de FTIR de comparação entre o óleo de girassol (OG) e o poliol de girassol (PG).

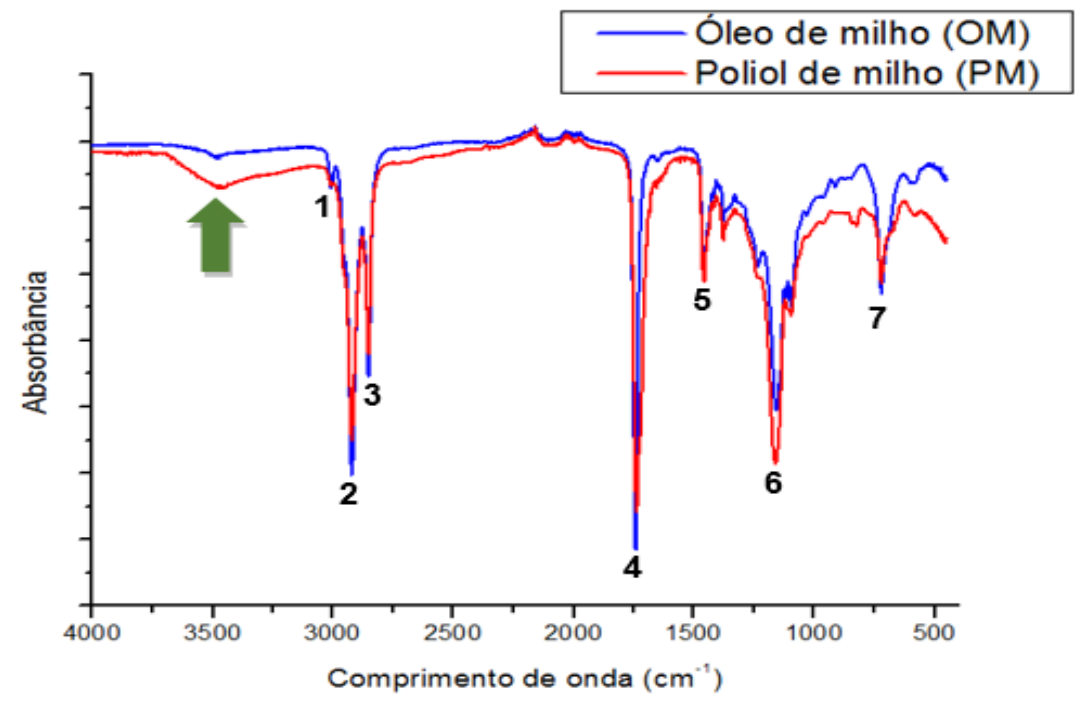

Figura 4: Espectro de FTIR de comparação entre o óleo de milho (OM) e o poliol de milho (PM).

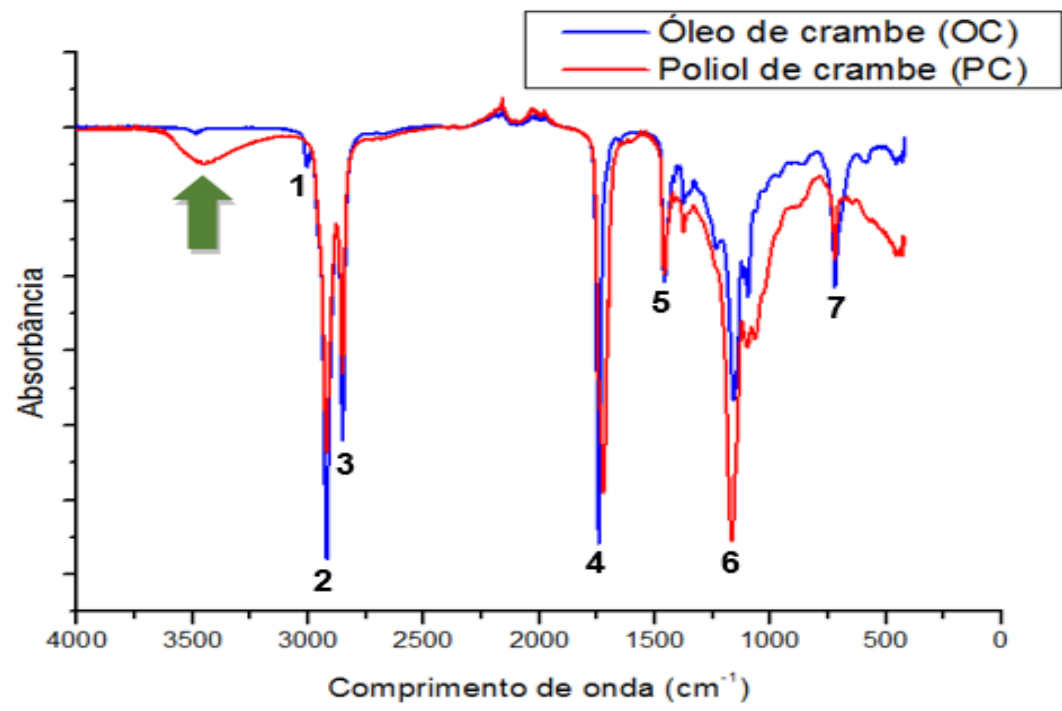

Figura 5: Espectro de FTIR de comparação entre o óleo de crambe (OC) e o poliol de crambe (PC). 
Ao observar os espectros dos óleos vegetais nas Figuras 3, 4 e 5, pode-se notar a semelhança dos mesmos e determinar o valor exato das bandas para análise das ligações presentes na molécula dos triacilglicerídeos.

Analisando os espectros e utilizando as tabelas da bibliografia BARBOSA [37], é possível fazer a atribuição das bandas na faixa de absorção que caracteriza o grupo alqueno com o estiramento da ligação C-H $\left(3100-3000 \mathrm{~cm}^{-1}\right)(1)$ de carbono hibridizado em $\mathrm{sp}^{2}$ proveniente de vibrações de olefinas, representando as insaturações presentes na cadeia carbônica; estiramento simétrico e assimétrico de ligação C-H $\left(3000-2850 \mathrm{~cm}^{-1}\right)$ (2) de grupos $\mathrm{CH}_{3}, \mathrm{CH}_{2}$ e $\mathrm{CH}$ de alcanos e grupos alquilas; estiramento da ligação C-H (2870-2840 cm-1) (3) característica de grupos $\mathrm{CH}_{2}$; a banda na faixa de (1810-1760 $\left.\mathrm{cm}^{-1}\right)$ característica do estiramento da banda de carbonila $(\mathrm{C}=\mathrm{O})(4)$, a banda na faixa de $\left(1600-1450 \mathrm{~cm}^{-1}\right)(5)$ proveniente de olefinas cis; a banda na faixa de $\left(1205-1125 \mathrm{~cm}^{-1}\right)$ (6) referente a deformação axial da ligação C-O de éster; e a banda em $721 \mathrm{~cm}^{-1}$ (7) que é comum em compostos com cadeia contendo mais de quatro grupos metilenos ligados, sendo referente à olefinas dissubstituídas [27, 29, 38, 39].

Há a predominância dos grupos alquilas e carbonilas nas moléculas constituintes do óleo e não é observada a presença de bandas características de grupamentos hidroxilas $\left(3400-3500 \mathrm{~cm}^{-1}\right)$ nos óleos vegetais.

Nos espectros dos polióis vegetais, nota-se o aparecimento de uma banda alargada na faixa de $3400-3500 \mathrm{~cm}^{-1}$, destacados com uma seta verde nas Figuras 3, 4 e 5. Essa banda caracteriza a presença do grupo hidroxila (OH) inserido na molécula do triglicerídeo [29, 34, 40].

Observa-se nos espectros dos polióis o desaparecimento da banda (1) na faixa de (3100-3000 $\left.\mathrm{cm}^{-1}\right)$, o que evidencia que parte das insaturações foram consumidas. Esse comportamento é justificado pela entrada das hidroxilas nas insaturações presentes nos ácidos graxos, demonstrando qualitativamente que as ligações $\mathrm{C}-\mathrm{H}$ de carbono hibridizado em $\mathrm{sp}^{2}$ proveniente das vibrações de olefinas foram quebradas e verificou-se o aparecimento da deformação axial da ligação O-H na faixa de (3400-3500 $\left.\mathrm{cm}^{-1}\right)$, referente a entrada das hidroxilas. Tal observação evidencia que o produto hidroxilado foi formado e que ainda há insaturações remanescentes da reação de hidroxilação, verificadas pela permanência da banda (5).

\section{CONCLUSÕES}

As análises dos índices de acidez (IA), índice de iodo (II) e densidade para os óleos vegetais estudados permitiram concluir que estes estavam em condições químicas e físicas para serem utilizados neste trabalho e para a síntese dos polióis vegetais.

Os resultados encontrados na caracterização dos polióis quanto aos índices de acidez e iodo foram coerentes com a literatura, uma vez que é verificado um aumento no IA devido a presença de ácidos graxos livres provenientes da hidrólise do óleo, e uma diminuição no II que ocorre pela reação de hidroxilação que inicia-se com o ataque do ácido às insaturações do óleo para inserção da hidroxila na molécula de triglicerídeo, e consequente obtenção do poliol.

Os espectros de FTIR dos polióis permitiram a identificação de bandas características de grupos hidroxilas na faixa de 3400-3500 $\mathrm{cm}^{-1}$. O poliol de girassol apresentou maior valor de $\mathrm{IOH}$ de 72,88 $\mathrm{mg}$ de $\mathrm{KOH} / \mathrm{g}$ de amostra e viscosidade de 1096,4 mPa.s. O poliol de crambe apresentou valor de $\mathrm{IOH}$ de 40,80 mg de KOH/g de amostra e viscosidade de 649,9 $\mathrm{mPa}$.s. O poliol de milho apresentou $\mathrm{IOH}$ de $37,40 \mathrm{mg}$ de $\mathrm{KOH} / \mathrm{g}$ de amostra e viscosidade de 486,8 $\mathrm{mPa}$.s.

Pode-se concluir que foi possível sintetizar os polióis vegetais a partir da reação de hidroxilação dos óleos estudados e os produtos obtidos podem ser usados para síntese de poliuretanos flexíveis e elastômeros, já que esses podem ser obtidos por polióis com baixos valores de índice de hidroxila ( $\mathrm{IOH}$ entre 28 e $160 \mathrm{mg}$ de $\mathrm{KOH} / \mathrm{g}$ ), aumentando-se o valor agregado do produto.

\section{AGRADECIMENTOS}

A Fundação MS pelo óleo de Crambe e ao CNPq pelo apoio financeiro.

\section{BIBLIOGRAFIA}

[1] PILLAI, P. K. S., LI, S., BOUZIDI, L., et al., "Metathesized palm oil \& novel polyol derivatives: Structure, chemical composition and physical properties", Industrial Crops and Products, v. 84, pp. 205-223, Jun. 2016.

[2] LligADAS, G., RONDA, J. C., GALIA, M., et al., "Plant Oils as Platform Chemicals for Polyurethane Synthesis: Current State-of-the-Art", Biomacromolecules, v. 11, pp. 2825-2835, Nov. 2010.

[3] LI, S., BOUZIDI, L., NARINE, S. S., "Polyols from self-metathesis-generated oligomers of soybean oil and their polyurethane foams", European Polymer Journal, v. 93, pp. 232-245, Aug. 2017. 
[4] ALLAUDDIN, S., SOMISETTI, V., RAVINDER, T., et al., "One-pot synthesis and physicochemical properties of high functionality soy polyols and their polyurethane-Urea coatings", Industrial Crops and Products, v. 85, pp. 361-371, Jul. 2016.

[5] REIZNAUTT, Q. B., GARCIA, I. T. S., SAMIOS, D., "Oligoesters and polyesters produced by the curing of sunflower oil epoxidized biodiesel with cis-cyclohexane dicarboxylic anhydride: Synthesis and characterization”, Materials Science and Engineering: C, v. 29, n. 7, pp. 2302-2311, Aug. 2009.

[6] ZLATANIC, A., LAVA, C., ZHANG, W., et al., "Effect of Structure on Properties of Polyols and Polyurethanes Based on Different Vegetable Oils", Journal of Polymer Science Part B-Polymer Physics, v. 42, n. 5, pp. 809-819, Mar. 2004.

[7] TAVARES, G. R., MASSA, T. B., GONÇALVES, J. E., et al., "Assessment of ultrasound-assisted extraction of crambe seed oil for biodiesel synthesis by in situ interesterification”, Renewable Energy, v. 111, pp. 659-665, Oct. 2017.

[8] HAZMI, A. S. A., AUNG, M. M., ABDULLAH, L. C., et al., "Producing Jatropha oil-based polyol via epoxidation and ring opening", Industrial Crops and Products, v. 50, pp. 563-567, Oct. 2013.

[9] FANG, Z., YANG, Z., JI, D., et al., "Novel synthesis of a soy-based polyol for a polyurethane rigid foam", RSC Advances, v. 6, n. 93, pp. 90771-90776, 2016.

[10] MOSIEWICKI, M. A., ARANGUREN, M. I., "A short review on novel biocomposites based on plant oil precursors", European Polymer Journal, v. 49, n. 6, pp. 1243-1256, Jun. 2013.

[11] PAUZI, N. N. P. N., MAJID, R. A., DZULKIFLI, M. H., et al., "Development of rigid bio-based polyurethane foam reinforced with nanoclay”, Composites Part B: Engineering, v. 67, n. 1, pp. 521-526, Dec. 2014.

[12] LI, J., JIANG, J., XU, J., et al., "Branched polyols based on oleic acid for production of polyurethane foams reinforced with bamboo fiber", Iranian Polymer Journal, v. 25, n. 9, pp. 811-822, Aug. 2016.

[13] SHIRKE, A. G., DHOLAKIYA, B. Z., KUPERKAR, K., "Modification of tung oil-based polyurethane foam by anhydrides and inorganic content through esterification process", Journal of Applied Polymer Science, v. 135, n. 5, pp. 1-10, Sep. 2017.

[14] COSTA, A. P. O., PETZHOLD, C. L., GERBASE, A. E., et al., "Síntese e Caracterização de Compósitos de Poliuretanas obtidas com Poliol-Soja/TDI/Cargas Minerais”, Revista Virtual de Química, v. 9, n. 4, pp. 1434-1448, Sep. 2017.

[15] SOTO, G. D., MARCOVICH, N. E., MOSIEWICKI, M. A., "Flexible polyurethane foams modified with biobased polyols: Synthesis and physical-chemical characterization", Journal of Applied Polymer Science, v. 133, n. 35, pp. 1-11, Sep. 2016.

[16] CHEN, R., ZHANG, C., KESSLER, M. R., "Polyols and Polyurethanes prepared from epoxidized soybean oil ring-opened by polyhydroxy fatty acids with varying $\mathrm{OH}$ numbers", Journal of Applied Polymer Science, v. 132, n. 1, pp. 41213-41223, Jan. 2015.

[17] SUN, L. J., YAO, C., ZHENG, H. F., et al., "A novel direct synthesis of polyol from soybean oil”, Chinese Chemical Letters, v. 23, n. 8, pp. 919-922, Aug. 2012.

[18] AUNG, M. M., YAAKOB, Z., KAMARUDIN, S., et al., "Synthesis and characterization of Jatropha (Jatropha curcas L.) oil-based polyurethane wood adhesive", Industrial Crops and Products, v. 60, pp. 177-185, Sep. 2014.

[19] VERONESE, V. B., MENGER, R. K., FORTE, M. M. C., et al., "Rigid Polyurethane Foam Based on Modified Vegetable Oil”, Journal of Applied Polymer Science, v. 120, n. 1, pp. 530-537, Apr. 2011.

[20] NASCIMENTO, B. Z., LIMA, K. F. F., SILVEIRA, V. C., et al., "Estudo da obtenção de poliol vegetal a partir da espécie Crambe abyssinica", Brazilian Journal of Production Engineering, v. 3, n. 1, pp. 27-34, 2017.

[21] CLEMENTE, M., ROCHA, R. J., IHA, K., et al., "Desenvolvimento de tecnologia de pré-polímeros na síntese de poliuretanos empregados em combustíveis sólidos”, Química Nova, v. 37, n. 6, pp. 982-988, Jul. 2014.

[22] LIDE, D. R., Handbook of Chemistry and Physics, 64 ed., CRC press, 1983.

[23] ANVISA, AGÊNCIA NACIONAL DE VIGILÂNCIA SANITÁRIA, http://portal.anvisa.gov.br/documents/10181/2718376/RES_482_1999_COMP.pdf/0b31ce35-6d43-42d6-8184-549de494987a?version=1.0. Acessado em abril de 2018.

[24] JASPER, S. P., BIAGGIONI, M. A. M., SILVA, P. R. A., "Caracterização físico-química do óleo e do biodiesel de Crambe abyssinica Hochst”, Revista Nucleus, v. 10, n. 2, pp. 183-190, Out. 2013.

[25] SILVA, S. A. Síntese e caracterização de poliuretanos - Espumas flexíveis puras e compósitos a partir de polióis à 
base de milho: e adesivos à base de derivados de óleo de rícino, Tese de D.Sc., UFPR, Curitiba, PR, Brasil, 2014.

[26] SiLVA, M. A. P., BIAGGIONI, M. A. M., SPEROTTO, F. C. S., et al., "Qualidade do óleo bruto de grãos de crambe (Crambe abyssinica Hochst) sob diferentes métodos de secagem”, Revista Energia na Agricultura, v. 28, n. 3, pp. 193-199, Jul. 2013

[27] MONTEAVARO, L. L., SILVA, E. O., COSTA, A. O., et al., "Polyurethane networks from formiated soy polyols: Synthesis and Mechanical Characterization", Journal of the American Oil Chemists Society, v. 82, n. 5, pp. 365-371, May. 2005.

[28] YAKUSHIN, V., STIRNA, U., BIKOVENS, O., et al., "Synthesis and Characterization of Novel Polyurethanes Based on Vegetable Oils Amide and Ester Polyols", Materials Science, v. 20, n. 3, pp. 277-282, 2014.

[29] SOUZA, V. H. R., SILVA, S. A., RAMOS, L. P., et al., "Synthesis and Characterization of Polyols Derived from Corn Oil by Epoxidation and Ozonolysis", Journal of the American Oil Chemists Society, v. 89, n. 9, pp. 1723-1731, Sep. 2012.

[30] KIRPLUKS, M., KALNBUNDE, D., BENES, H., et al., "Natural oil based highly functional polyols as feedstock for rigid polyurethane foam thermal insulation”, Industrial Crops and Products, v. 122, pp. 627-636, Oct. 2017.

[31] ABREU, D. H. S., Craqueamento termocatalítico da borra de neutralização do óleo de palma (Elaeis guineenses) em escala piloto, Dissertação de M.Sc., UFPA, Belém, PA, Brasil, 2013.

[32] SANTOS, J. C., SANTOS, I. M. G., SOUZA, A. G., "Effect of heating and cooling on rheological parameters of edible vegetable oils", Journal of Food Engineering, v. 67, n. 4, pp. 401-405, Apr. 2005.

[33] VAZ, E. L. S., ACCIARI, H. A., ASSIS, A., et al., "Uma experiência didática sobre viscosidade e densidade", Química Nova na Escola, v. 34, n. 3, pp. 155-158, Aug. 2012.

[34] KONG, X., LIU, G., CURTIS, J. M., "Novel polyurethane produced from canola oil based poly (ether ester) polyols: Synthesis, characterization and properties", European Polymer Journal, v. 48, n. 12, pp. 2097-2106, Dec. 2012.

[35] JORGE, N., Química e tecnologia de óleos vegetais, São Paulo, Cultura Acadêmica, 2009.

[36] CARRIÇO, C. S., "Obtenção de espumas de poliuretano a partir de coprodutos da cadeia dos biocombustíveis e resíduos agroindustriais”, Tese de D.Sc., UFMG, Belo Horizonte, MG, Brasil, 2017.

[37] BARBOSA, L. C. A., Espectroscopia no Infravermelho na caracterização de compostos orgânicos, Viçosa, Editora UFV, 2007.

[38] PETROVIC, Z. S., CVETKOVIC, I., HONG, D., et al., "Polyester polyols and polyurethanes from ricinoleic acid", Journal of Applied Polymer Science, v. 108, n. 2, pp. 1184-1190, Apr. 2008.

[39] LOZADA, Z., SUPPES, G. J., TU, Y., et al., "Soy-Based Polyols from Oxirane Ring Opening by Alcoholysis Reaction”, Journal of Applied Polymer Science, v. 113, n. 4, pp. 2552-2560, Aug. 2009.

[40] OMRANI, I., FARHADIAN, A., BABANEJAD, N., et al., "Synthesis of novel high primary hydroxyl functionality polyol from sunflower oil using thiol-yne reaction and their application in polyurethane coating", European Polymer Journal, v. 82, pp. 220-231, Sep. 2016.

\section{ORCID}

Bárbara Zon Nascimento

Ana Paula Oliveira Costa
http://orcid.org/0000-0001-8768-078X

https://orcid.org/0000-0003-0699-9997 